\title{
TEMA -2018: Diagnóstico y Manejo de la Primera Convulsión
}
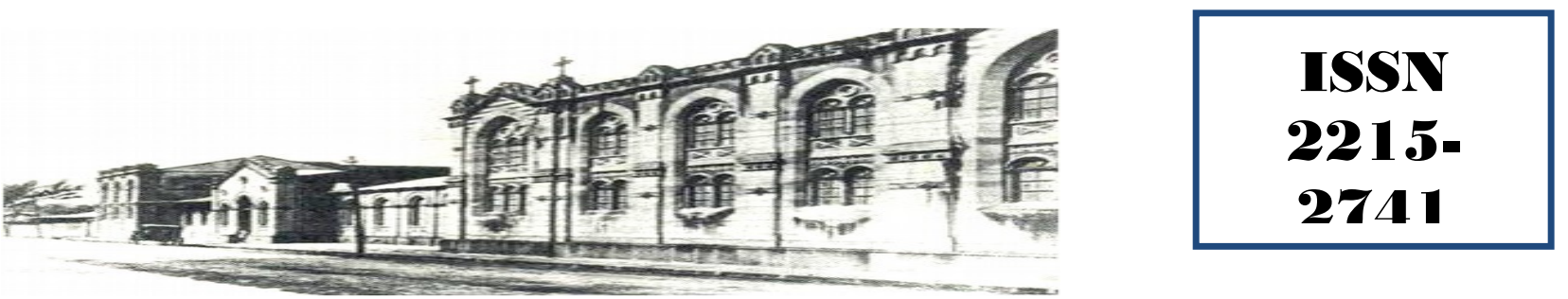

Hospital San quan de Dias, San Jasé, Casta Rica. Fundada en 1845

Recibido:

Aceptado:

$19 / 01 / 2018$

$15 / 03 / 2018$

Carlos Mario Sequeira Quesada ${ }^{1}$ José Chang Segura ${ }^{2}$

${ }^{1}$ Médico General. Graduado de la Universidad de Costa Rica. carlos.sequeiraquesada@ucr.ac.cr

${ }^{2}$ Médico Residente en la Especialidad de Neurología. Hospital Calderón Guardia.

\section{RESUMEN}

Una convulsión se define como actividad eléctrica cerebral anormal excesiva e hipersincrónica que genera síntomas neurológicos transitorios, los cuales deben ser diferenciados al momento de su presentación de entre una amplia gama de otros diagnósticos neurológicos y no neurológicos. Las convulsiones deben ser clasificadas entre focal y general, sintomática aguda y sintomática provocada y entre provocadas y no provocadas. No todo paciente que convulsiona volverá a hacerlo. Al paciente con convulsión se le debe realizar un electroencefalograma y una resonancia magnética. El riesgo de recaída en un paciente con una primera convulsión no provocada es de $36 \%$ en el primer año y de $45 \%$ en el segundo. Aquellos que presentan alteraciones corticales en estudios de imágenes o actividad epileptiforme en el electroencefalograma sin embargo eleven este riesgo hasta $60 \%$. El tratamiento con fármacos anticonvulsivantes debe ser iniciado en pacientes que cumplen la definición de epilepsia y discutido en el paciente con una única convulsión no provocada. Posterior a 2 años sin crisis convulsivas la suspensión de dicho tratamiento puede ser valorada.

\section{PALABRAS CLAVE}

Convulsión. Epilepsia. Paroxístico. Antiepiléptico. Recurrencia

\section{ABSTRACT}

A seizure is defined as excesive and hypersynchronic electric brain activity that generates transitory neurologic symptoms, which must be differentiated among a wide range of potential neurologic and non-neurologic diagnosis. Seizures must be classified as focal or generalized, acute symptomatic or remote symptomatic and provoked and unprovoked. Not all patients that have a seizure will do so again. Every patient with a convulsive episode must receive an electroencephalogram and a magnetic resonance. The risk of recurrence after a first unprovoked seizure is $36 \%$ during the first year 
and $45 \%$ during the second year. Those who present with cortical abnormalities in image studies and epileptic activity in electroencephalogram will elevate this risk up to $60 \%$. Treatment with antiepileptic drugs must be initiated in those patients that fulfill criteria for epilepsy and discussed in those with a single unprovoked seizure. After 2 years free of crisis discontinuation of drug therapy can be considered.

\section{KEY WORDS}

Seizure. Epilepsy. Paroxysmal. Antiepileptic. Recurrent

\section{INTRODUCCIÓN}

La aparición de una convulsión a lo largo de la vida de las personas es un evento relativamente frecuente, calculándose que cerca del $10 \%$ de la población tendrá una de ellas. Se estima que 150 000 adultos experimentan anualmente su primera convulsión y esto tan solo considerando los Estados Unidos.

Aunque sea un único episodio, haber convulsionado resulta un evento traumático tanto física como psicológicamente, y conlleva muchas dudas tanto diagnósticas como terapéuticas. Las convulsiones recurrentes presentan un problema aún más serio y económicamente costoso.

La recidiva de las convulsiones sin embargo no ostenta una frecuencia similar, estimándose que menos de la mitad de dichas personas tendrán múltiples episodios convulsivos. De aquí se desprende la necesidad e importancia de utilizar términos correctos, puesto que el presentar una convulsión no es lo mismo que ser diagnosticado con epilepsia, y etiquetar a un paciente con este último término es adjuntarle todo el estigma social que el mismo conlleva ${ }^{1,2,3}$

El término convulsión, a pesar del uso que tiende a dársele en la población general, hace referencia a episodios paroxísticos y episódicos de actividad eléctrica cerebral anormal, excesiva e hipersincrónica resultando en signos $\mathrm{y} / \mathrm{o}$ síntomas transitorios visuales, motores, somatosensoriales o de comportamiento. Las convulsiones además pueden y deben ser clasificadas de diferentes maneras, categorizándose según la nomenclatura propuesta por la Liga Internacional contra la Epilepsia (ILAE) en focales, generalizadas o desconocidas. La ILAE también propone una distinción de las convulsiones según su etiología, punto el cual será ampliado más adelante. Además, de la mano con la etiología subyacente, las convulsiones pueden ser separadas en provocadas y no provocadas. Las convulsiones provocadas son aquellas debidas a causas identificables como medicamentos, drogas de abuso o causas metabólicas. Por otro lado, las convulsiones que resultan de procesos cerebrales agudos como por ejemplo la encefalitis, se les llama sintomáticas agudas.

Las convulsiones sintomáticas agudas pueden ser clasificadas junto a las provocadas, sin embargo, ciertos autores recomiendan entenderlas como grupos separados. Es relevante remarcar que ninguna de ellas es contemplada dentro la definición de epilepsia. Por otro lado, existen las ya mencionadas convulsiones no provocadas, las cuales son episodios que ocurren en ausencia de un factor precipitante y que pueden ser a su vez separadas en dos subgrupos, las convulsiones debidas a una etiología desconocida y aquellas, que, por default, su causa si es identificada.

Aquellas que se deben a una anormalidad cerebral persistente estática se les llama convulsiones sintomáticas remotas, y las que se propician por lesiones progresivas son las convulsiones sintomáticas progresivas. Las convulsiones no provocadas difieren de las agudas sintomáticas tanto en riesgo de recurrencia como mortalidad, y conceptualmente se deben entender las sintomáticas agudas como diferentes a las sintomáticas remotas ${ }^{1,3,4,5,6,7}$.

La epilepsia, por otra parte, no es una única entidad, sino una colección de desórdenes que tienen en común la ocurrencia de convulsiones, hacienda mención a un fenómeno clínico y es definida por la ILAE como por lo menos dos convulsiones no provocadas ocurriendo con 24 horas de separación entre cada una, o como una única convulsión no provocada con una probabilidad de recidiva similar al riesgo de recurrencia general que se presenta posterior a dos convulsiones no provocadas en los 
subsecuentes 10 años, o por último, el diagnóstico de un síndrome epiléptico.

Esta definición implica que una persona con una única convulsión o inclusive múltiples convulsiones provocadas por una causa corregible o evitable no tiene necesariamente epilepsia. Los componentes de esta definición serán ampliados a lo largo de este artículo ${ }^{3,6,7,8}$.

Las decisiones sobre el tratamiento de una única primera convulsión incluyen consideraciones sobre la probabilidad de una segunda convulsión no provocada, las consecuencias de presentarla, la eficacia de la medicación en prevenir futuras convulsiones y su potencial toxicidad. Realizar una valoración precisa de una primera convulsión evita el tratamiento farmacológico innecesario en pacientes que tienen pocas probabilidades a presentar una segunda convulsión no provocada. A lo largo de esta revisión, abordaremos el abordaje diagnóstico de una convulsión, el riesgo de recurrencia de convulsiones subsecuentes según el escenario del paciente y el momento adecuado para iniciar tratamiento farmacológico ${ }^{3}$.

\section{DISCUSIÓN}

\section{Diagnóstico Diferencial de Signos y Síntomas Paroxísticos}

Como fue mencionado, la convulsión es un episodio transitorio y paroxístico, sin embargo, como resulta lógico, no es el único diagnóstico que concuerda con esta descripción. El abordaje de una primera convulsión y la decisión de brindar o no tratamiento según el riesgo estimado de recurrencia se basa precisamente en que el médico tratante haya hecho un diagnóstico certero de convulsión, diferenciando a la misma de múltiples imitadores ${ }^{4,5}$.

Una historia de previas convulsiones apoya el diagnóstico de epilepsia e indica un riesgo subyacente mayor de recurrencias, sin embargo, si se trata de la primera convulsión del paciente evidentemente este dato no estará presente. Se desprende que la historia clínica es de vital importancia en este abordaje, así como una examinación tanto física como neurológica al momento de presentación inicial.
Si la historia es lo suficientemente característica se puede llegar al diagnóstico de convulsión epiléptica sin necesidad de pruebas diagnósticas o de laboratorio. Sin embargo, es frecuente que surjan dudas debido a las similitudes entre este diagnóstico y muchas otras entidades. Se debe mencionar que no existe ninguna prueba, hallazgo clínico o síntoma que por sí mismo de manera confiable pueda discriminar entre una convulsión epiléptica y otro evento paroxístico. Además de esto, el testimonio de un testigo tampoco es infalible debido a que su recolección de los hechos puede ser variable ${ }^{2}$.

El diagnóstico diferencial es amplio, debido a que múltiples desórdenes paroxísticos pueden imitar una convulsión, simulándola en aspectos tales como características fenotípicas, duración, características postictales e inclusive hallazgos electroencefalográficos.

Los llamados fenómenos no epilépticos son episodios de alteración en el movimiento, la sensación y la experiencia que se distinguen de una convulsión epiléptica por su falta de asociación con anormalidades eléctricas ictales. De un modo general, los fenómenos no epilépticos pueden dividirse en dos grandes grupos: entidades fisiológicas y entidades psicogénicas. A su vez, el cajón de entidades fisiológicas es subseparado en patologías neurológicas y no neurológicas. Los eventos psicogénicos son una fuente frecuente de confusión y son fenómenos similares a la convulsión, pero faltándole las características clínicas y electrofisiológicas de una convulsión verdadera.

Las entidades a considerar en el diagnóstico diferencial de síntomas transitorios paroxísticos no serán profundizadas una a una, sin embargo, a modo de generalización se puede decir que un inicio repentino de disfunción neurológica focal sin aviso sugiere etiología vascular, la progresión lenta de los síntomas durante unos segundos sugiere fenómeno ictal mientras que aquella progresión a lo largo de minutos u horas hace sospechar en una diátesis migrañosa. Existen sin embargo excepciones a estas afirmaciones. Más entidades se encuentran enumeradas en Tabla 1, dejándose por fuera a la categoría de trastornos funcionales o psicogénicos así como muchas 
otras de la grandísima gama de patologías a considerar en este contexto clínico $4,5,10,11,12,13,14$.

Este último grupo, sin embargo, es de gran importancia. En centros de atención especializados en epilepsia, hasta $88 \%$ de los pacientes con fenómenos no convulsivos son eventualmente diagnosticados con una etiología psicogénica como la explicación de su cuadro. El diagnóstico de estos fenómenos psicogénicos se realiza basándose en toda la evidencia a disposición, incluyendo historia clínica, examen físico, la descripción y visualización si fuese posible de los eventos paroxísticos, así como los hallazgos (o falta de) en EEG ictal e interictal.

No existe un único punto en el abordaje clínico que por sí solo sea patognomónico, lo cual dificulta grandemente el diagnóstico. Sin embargo, retrasar el diagnóstico de convulsiones psicogénicas empeora el pronóstico del paciente. Se favorece actualmente un abordaje integral e integrado, lo cual podría ayudar en el diagnóstico, y evidencia reciente apunta que la terapia cognitiva pudiese ser de utilidad (ver tabla 2) ${ }^{15}$.

Tabla 1: Diagnóstico Diferencial de Síntomas y Signos Neurológicos paroxísticos

\begin{tabular}{|c|c|}
\hline Diagnósticos Neurológicos & $\begin{array}{l}\text { Diagnósticos No } \\
\text { Neurológicos }\end{array}$ \\
\hline 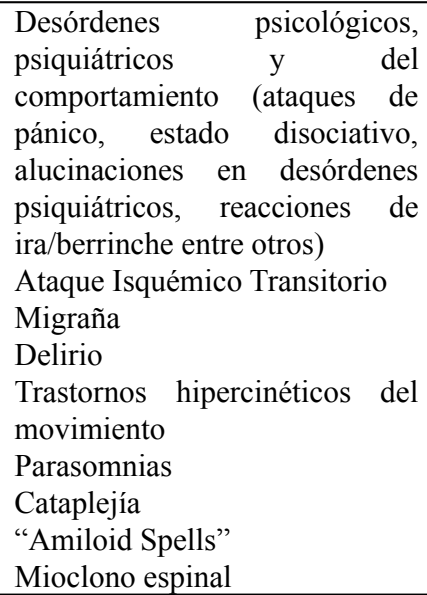 & $\begin{array}{l}\text { Síncope vasovagal } \\
\text { Hipotensión } \\
\text { ortostática } \\
\text { Convulsiones } \\
\text { anóxicas reflejas } \\
\text { Valsalva convulsiva } \\
\text { Obstrucción } \\
\text { impuesta de vía aera } \\
\text { Síncope cardíaco } \\
\text { Ataques } \\
\text { hipercianóticos } \\
\text { Síndrome } \\
\text { Sandifer de }\end{array}$ \\
\hline
\end{tabular}

Tabla 2: Diferencias entre crisis convulsivas y crisis psicogénicas o pseudocrisis

\begin{tabular}{|c|c|c|}
\hline Signos & $\begin{array}{c}\text { Hallazgos a la } \\
\text { examinación }\end{array}$ \\
\hline
\end{tabular}

\begin{tabular}{|c|c|c|}
\hline $\begin{array}{c}\text { Convulsiones } \\
\text { psicogénicas } \\
\text { no } \\
\text { epilépticas }\end{array}$ & $\begin{array}{l}\text { Larga duración, curso } \\
\text { fluctuante, } \\
\text { movimientos } \\
\text { asincrónicos, } \\
\text { movimientos pélvicos, } \\
\text { movimientos de } \\
\text { cabeza de lado a lado, } \\
\text { cierre de ojos ictal, } \\
\text { llanto/sollozo ictal, } \\
\text { recuerdos activos del } \\
\text { supuesto período de } \\
\text { no respuesta }\end{array}$ & $\begin{array}{l}\text { Resistencia a } \\
\text { la apertura } \\
\text { palpebral, } \\
\text { protección } \\
\text { activa de } \\
\text { recibir golpes } \\
\text { a la cara, } \\
\text { evidencia de } \\
\text { fijación visual. }\end{array}$ \\
\hline $\begin{array}{l}\text { Convulsiones } \\
\text { Epilépticas }\end{array}$ & $\begin{array}{l}\text { Ocurrencia durante } \\
\text { sueño confirmado por } \\
\text { EEG, } \\
\text { confusión/obtundació } \\
\text { n postictal, respiración } \\
\text { dificultosa postictal. }\end{array}$ & $\begin{array}{l}\text { Mordeduras } \\
\text { linguales } \\
\text { laterales } \\
\text { severas, } \\
\text { reflejo corneal } \\
\text { perdido, } \\
\text { respuesta } \\
\text { plantar } \\
\text { extensora }\end{array}$ \\
\hline
\end{tabular}

Fuente: Diagnosis and Treatment of Nonepileptic Seizures. 2016

\section{Abordaje de la Primera Convulsión}

Como fue previamente indicado, el primer obstáculo al cual el médico debe enfrentarse es identificar el evento como una convulsión de entre todos los diagnósticos diferenciales posibles. El diagnóstico del tipo de convulsión y el síndrome epiléptico se basa en la descripción del comportamiento de la convulsión, así como de las manifestaciones en la electroencefalografía, auxiliado con neuroimágenes e investigaciones genéticas en algunos casos, y es importante tener claro los pasos para realizarlo apropiadamente debido a las serias implicaciones que conlleva el mismo para los pacientes y sus familias 4 .

Desde el momento en que el paciente es diagnosticado con una convulsión, el clínico debe dirigir sus esfuerzos a determinar la etiología del cuadro. Las diversas posibilidades etiológicas pueden ser encajonadas en seis diferentes grupos: estructural, genética, infecciosa, metabólica, inmune y desconocida. Se debe acotar que estas categorías no son mutuamente excluyentes, por lo que la convulsión/epilepsia de un paciente puede ser clasificada dentro de varias de estas categorías a la vez. 
Los ya citados estudios imagenológicos y el electroencefalograma (EEG) son útiles para este ejercicio diagnóstico, pero a su vez otros estudios ancilares pueden ser necesarios para establecer la entidad catalizante de la convulsión. Entre ellos podemos citar diferentes exámenes como glucemia, hemograma completo, tamizaje toxicológico y un panel electrolítico, con énfasis en el sodio. Las guías indican sin embargo que si bien es cierto estas pruebas pueden resultar útiles, no existe información suficiente para recomendarlas como pruebas de rutina a todos los pacientes en su primera convulsión. La punción lumbar presenta una condición similar, no siendo necesaria protocolariamente para todos los pacientes, pero resultando útil en un paciente febril. Todo lo anterior indica que todos y cada uno de los pacientes en su primera convulsión necesitan un EEG y un estudio de imágenes.

Cualquier prueba adicional debe seleccionarse según el contexto de cada paciente. Otra prueba mencionada dentro del posible abordae diagnóstico de estos pacientes es la realización de un electrocardiograma, dirigido a la detección de una prolongación de QT. Este resulta de simple obtención en la mayoría de servicios de emergencia donde estas personas serán más frecuentemente abordadas inicialmente. ${ }^{4,5,11}$

$\mathrm{Y}$ es que una vez que se ha identificado el fenómeno paroxístico como una convulsión, el siguiente paso que el médico debe realizar es catalogar la convulsión como focal (actividad eléctrica involucra redes neurales confinadas a un único hemisferio), generalizada (actividad eléctrica comienza de manera bilateralmente distribuida en redes neuronales de ambos hemisferios desde el inicio) o desconocida. El comportamiento ictal específico debe ser descrito, lo cual ayuda a subdividir todavía más las convulsiones.

De esta manera, las convulsiones focales pueden presentar (focales con síntomas discognitivos o de desconexión) o no pérdida de la conciencia. Esta última es a su vez denominada motora, autonómica o sensorial objetiva según sus componentes fenotípicos observables. A su vez, las convulsiones generalizadas abarcan las ausencias típicas y atípicas, incluyendo aquellas ausencias con características especiales, mioclonías, convulsiones clónicas, tónicas, tónico-clónicas y atónicas. La última categoría de convulsiones, la denominada desconocida, incluye a los espasmos epilépticos ${ }^{4,517}$.

El énfasis para pacientes con su primera convulsión es entonces el diagnóstico expedito y la evaluación para determinar la etiología subyacente. Pruebas diagnósticas pueden ser de utilidad para determinar el diagnóstico y el pronóstico para futuras recurrencias. Posterior a una historia clínica detallada y exhaustiva tomada del paciente y familiares o testigos, la investigación con EEG y neuroimágenes se vuelve esencial. Esto último toma importancia principalmente cuando se extrapola al contexto de definir cuales pacientes pasarán a ser catalogados como epilépticos, esto según su riesgo de recurrencia.

El aspecto de riesgo de recidivas será ampliado en una sección subsecuente, sin embargo, en este momento debe ser mencionado en relación con la definición brindada de epilepsia, y es que se había establecido que por epilepsia puede entenderse una única convulsión no provocada pero que ocurre en un paciente con el mismo riesgo de recaída a un plazo de 10 años que aquél paciente que ya ha presentado previamente dos convulsiones. Este riesgo es de aproximadamente $60 \%$, y en un paciente con una primera convulsión es alcanzado según lo documentado en la resonancia magnética y el EEG.

En estos estudios se debe buscar lesiones corticales como displasia cortical focal, tumores primarios o metastásicos y un infarto cerebral antiguo entre otros, esto en los estudios de imágenes, así como fenómenos epileptogénicos en el electroencefalograma (complejos de espigaonda puntiagudos, descargas periódicas lateralizantes o generalizadas, enlentecimiento focal, actividad rítmica delta temporal, hipsiarritmias, supresión de estallido y descargas espiga-onda continuas) ${ }^{4,18,19,20}$.

La electroencefalografía es entonces el estudio diagnóstico más comúnmente realizado en los abordajes diagnósticos de personas con convulsiones/epilepsia. Esta herramienta ha aumentado nuestro entendimiento de la patología de las convulsiones y se ha convertido en un arma invaluable, y sus aplicaciones clínicas se extienden no solo al diagnóstico de epilepsia, 
sino también en seleccionar la terapia anticonvulsivante, evaluar la respuesta a dicha farmacoterapia, determinar cuáles pacientes pudieran ser candidatos a suspensión del tratamiento, así como una ayuda en la localización quirúrgica.

La Academia Americana de Neurología (AAN) recomienda el EEG para el diagnóstico de epilepsia tanto en adultos como en niños, con inclusion de estimulación fótica, hiperventilación y deprivación del sueño como parte del protocolo fungiendo como procedimientos de activación. Un patrón epileptiforme de los previamente citados en el contexto de una primera convulsión predice una recurrencia de $30 \%$ a $70 \%$. Sin embargo, se debe resaltar que la sensibilidad de un único EEG para detector una anormalidad epileptiforme puede de ser de hasta menos de $50 \%$ en personas con epilepsia. EEGs repetidos pudieran ser entonces de importancia, aumentando dicha sensibilidad hasta 80 a $90 \%$. Igual de importante resulta el tiempo de separación entre el evento paroxístico y la realización del estudio.

Las guías recomiendan que este se lleve a cabo entre 24 y 48 horas posterior a la primera convulsión, sin embargo, diversos estudios han concluido que entre más pronto se realice el estudio, aumenta la probabilidad de documentar las descargas epileptogénicas. Se ha recomendado que sería más apropiado realizarlo en las primeras 12 horas post-convulsión. Por otro lado, la resonancia magnética ostenta un rol en el abordaje del paciente por su habilidad de identificar hallazgos patológicos asociados al cuadro clínico, determinar zonas epileptogénicas y eventualmente determinar objetivos quirúrgicos. Hasta 29\% de los pacientes con su primera convulsión tendrán anormalidades en este estudio de imágenes, y hasta $12 \%$ podrían presentar dichas alteraciones aun contando con una tomografía cerebral normal ${ }^{4,21}$.

Al valorar una supuesta primera convulsión, el médico tratante debe determinar si es en efecto la primera vez que este fenómeno ocurre, o si por lo contrario ya se ha presentado en el pasado múltiples veces. Una historia clínica cuidadosa podrá esclarecer lo anterior, en especial para aquellas convulsiones focales con desconexión o las ausencias infantiles típicas. Si el paciente en efecto ha tenido convulsiones previamente, es importante entonces determinar cuando ocurrieron estas a lo largo del tiempo, esto por cuanto el riesgo de recurrencia se modifica según la cantidad de convulsiones haya presentado el paciente ${ }^{3}$.

\section{Riesgo de Recurrencia}

Las convulsiones no provocadas pueden ser únicas o recurrentes, como el párrafo anterior implica, y ambas con diferentes probabilidades de presentar recaídas. Aunque todos los pacientes con una convulsión no provocada potencialmente podrían tener epilepsia, la recurrencia en sus cuadros convulsivos es observada únicamente en $50 \%$. Diferentes estudios y trabajos han abordado este aspecto, y han establecido si bien es cierto 1 de cada 10 personas convulsionarán en algún momento de la vida, el riesgo de recaída en un plazo de 1 año posterior a una primera y única convulsión es de 36 a 37\%, aumentando a $45 \%$ (probabilidad oscila entre 21 y $45 \%$ ) a los 2 años y manteniéndose en cifras similares a los 5 años. Estos datos sin embargo se incrementan si el paciente presenta dos convulsiones no provocadas.

Una vez que esto suceda, su riesgo de recidiva se dispara un $73 \%$ de tener un tercer episodio y en $76 \%$ el de tener un cuarto. Este aumento de recurrencia posterior la segunda convulsión es el que se incorpora a la definición dada por la ILAE para justamente fundamentar su concepto. La mayoría de recaídas ocurren en los primeros 365 días. Establecer la etiología atrás de la convulsión es también importante, puesto que las llamadas convulsiones sintomáticas (estructurales o metabólicas), si ya han ocurrido dos veces en el paciente, presentan un $87 \%$ a los 5 años de ocurrir por tercera vez, esto frente a un $64 \%$ en el mismo contexto, pero con epilepsias idiopáticas o criptogénicas ${ }^{3,22,23}$.

Como fue mencionado, es importante realizar la identificación de cuales convulsiones son provocadas, y más específicamente, cuales pertenecen al grupo de sintomáticas agudas. Diferentes estudios, en los cuales definen a las convulsiones sintomáticas agudas como aquellas que ocurren a los siete días de haberse presentado un evento cerebrovascular o traumacraneoencefálico y durante una infección 
activa de sistema nervioso central, encontraron una mortalidad 8.9 veces mayor a la ostentada por un paciente con una primera convulsión no provocada en los primeros 30 días, sin embargo, con el tiempo la mortalidad entre ambos grupos se equipara.

los 10 años, los pacientes con una convulsión provocada tuvieron un $80 \%$ menos de probabilidad de experimentar una segunda convulsión. Estos datos fundamentan el concepto que si bien es cierto la mortalidad temporalmente puede ser mayor en las agudas sintomáticas, el riesgo de recaída es mucho menor, por lo que estos pacientes no se consideran con epilepsia. Estos números además son los que llevan a la AAN a juntar las convulsiones agudas sintomáticas con las convulsiones provocadas dentro de un mismo grupo, en el sentido de no brindarles tratamiento antiepiléptico a priori, como se ampliará a continuación.

Sin embargo, se debe recalcar que las convulsiones sintomáticas agudas podrían ser separadas de las susodichas convulsiones provocadas, particularmente aquellas propiciadas por problemas metabólicos, medicamentosos o por drogas, debido a que las sintomáticas agudas podrían causar eventualmente lesiones cerebrales y cambios crónicos, mientras que esto no se presenta con todas las causas de convulsiones provocadas ${ }^{3}$.

Además de los hallazgos en EEG y resonancia magnética ya discutidos y su rol como factores que incrementan la posibilidad de convulsiones recidivantes, los estudios documentan que otro factor con este mismo efecto es el momento en que ocurran dichas convulsiones. Las convulsiones nocturnas se asocian con un incremento de 2.1 en el riesgo en comparación con los eventos que ocurren en pacientes despiertos.

Por otro lado, otros factores que no se asociaron con un incremento en el riesgo de recurrencia después de una primera convulsión no provocada son la edad del paciente, el sexo, la historia familiar de convulsiones, el tipo de convulsiones o la presentación con estatus epiléptico o múltiples convulsiones discretas con 24 horas de separación entre sí y recuperación completa entre ellas ${ }^{1,23,24}$.

\section{Tratamiento Farmacológico}

Una vez considerado todo lo anterior, el médico abordando el paciente con su primera convulsión deberá realizar la decisión de brindar o no brindar tratamiento con drogas anticonvulsivantes. Para tomar esta decisión, diferentes factores deben ser tomados en cuenta, la mayoría de ellos en relación a la caracterización de la convulsión presentada por el paciente. El médico tratante debe tomar en cuenta si la convulsión es provocada o no provocada, si la misma es focal o generalizada, y dentro de ellas a cuál subdivisión fenotípica pertenece. Como fue además previamente abordado, se debe además tomar en cuenta si la convulsión corresponde a una sintomática aguda o remota. Una tangible que hasta la fecha no ha sido completamente resuelto si tiene o no un rol en la selección de iniciar o no farmacoterapia es la edad del paciente. Se sabe sin embargo que el tipo de convulsión más frecuente sí varía según el grupo etario, y para escoger el fármaco específico a utilizar se debe incluir en la toma de decisiones el estilo y situación de vida del paciente $^{3}$.

En el contexto sin embargo de un paciente en su primera convulsión, el factor más importante en el momento de guiar la decisión de brindar o no tratamiento farmacológico es el riesgo de recurrencia del paciente, previamente comentado, y la habilidad de dicho fármaco de disminuir esas posibles recidivas.

Esta habilidad de disminuir recurrencias ha sido estudiada por diferentes ensayos clínicos centrándose principalmente en el período de los dos años posteriores a dicha primera convulsión, esto pues como fue mencionado, es el rango de tiempo donde ocurren con más frecuencia las recaídas. La mayoría de la evidencia con la que se cuenta al respecto en la actualidad y sobre la cual se han fundamentado las guías de manejo más recientes proviene principalmente de los estudios FIR.S.T y MESS.

Estos y otros estudios de menor renombre han fundamentado la conclusión dada por las guías de la AAN de que el tratamiento inmediato con fármacos antiepilépticos después de la primera convulsión reduce el riesgo de recaídas en hasta $35 \%$, sin embargo, no se observa ningún 
beneficio en la calidad de vida del paciente. Además, otros ensayos clínicos han concluido que dicho tratamiento inmediato en contraposición a retrasar el inicio de la farmacoterapia no genera un aumento en la recurrencia de convulsiones si se sigue al paciente en un período de tiempo más largo $(3 \mathrm{o}$ más años) ${ }^{1,3,25,26}$.

Agregado a la habilidad o no de disminuir episodios paroxísticos convulsivos por parte de cada fármaco antiepiléptico, se debe considerar además los efectos adversos que dichos esquemas terapeúticos pueden acarrear. Se estima que la incidencia de efectos adversos en la población que se viene describiendo oscila entre los 7 a 31\%. Dichos efectos adversos tienden a ser leves y reversibles al realizar un cambio a un otro medicamento anticonvulsivante ${ }^{1,3}$.

La información previa es la que fundamenta las recomendaciones de la AAN respecto a la farmacoterapia de la primera convulsión, las cuales son enfocadas principalmente en la comunicación médico-paciente. Se le debe informar al paciente de su riesgo exacto de presentar una segunda convulsión, y que esta posibilidad es mayor en los primeros dos años postevento. Se le debe enfatizar al paciente en cuales factores de riesgo pudiesen aumentar dicho riesgo (así como ponderar cuales pacientes según dichos factores de riesgo pasarían a definirse como epilépticos).

Se debe ser enfático, sin embargo, y referente al fármaco anticonvulsivante, que si bien es cierto la medicación disminuye la probabilidad que vuelva a convulsionar, esto no es equivalente a decir que la droga hará imposible que otro evento como el primero ocurra; el fármaco no jugará ningún rol en mejorar la calidad de vida del paciente y no son sustancias inocuas, teniendo efectos adversos reales, si bien es cierto leves y reversibles su gran mayoría.

Se debe acotar además que todo paciente que cumpla con la definición brindada para la epilepsia debe ser iniciado en tratamiento anticonvulsivante, pues en ellos los objetivos terapeúticos van más allá solo disminuir la recurrencia de convulsiones. Por otro lado, las convulsiones no provocadas y las sintomáticas agudas per se no van a requerir tratamiento anticonvulsivante, sino enfocarse en el abordaje de la entidad subyacente que propició la convulsión ${ }^{1,3}$.

Lo anterior no se debe interpretar como una justificación de que la farmacoterapia debe ser negada a un paciente posterior a una única convulsión no provocada sin hallazgos de relevancia en el EEG y resonancia magnética. La decisión de iniciar tratamiento o no debe ser individualizada a cada paciente y analizada caso por caso.

Un paciente puede tener una ocupación laboral en la cual convulsionar pudiera no ser aceptado, por lo cual, aunque no influya en su calidad de vida y conlleve ciertos riesgos, la disminución de convulsiones en el período de mayor recaídas podría ser suficiente para el paciente y llevarlo a iniciar tratamiento. El rol del médico es conocer la información, y la evidencia detrás de instaurar, y no instaurar, tratamiento farmacológico, y de esa forma tomar junto al paciente una decisión conjunta ${ }^{1,3,16}$.

De haberse tomado la decisión de iniciar tratamiento anticonvulsivante, eventualmente puede surgir en el paciente la interrogante de si es posible - y cuando- suspender el tratamiento. Muchas veces el profesional de salud presenta más temores al respecto que el mismo paciente, sin embargo, la lenta suspensión del anticonvulsivante es una opción real.

Dicho acto es más factible en ciertos pacientes que en otros. Un inicio de las crisis entre los 2 y 11 año, una mentación normal, una etiología de las crisis idiopáticas, un examen neurológico sin hallazgos, haber respondido de manera satisfactoria al tratamiento farmacológico y tener en el momento de consideración bajos niveles séricos del mismo, así como un período libre de crisis mayor a 2 años son todos factores favorables para la descontinuación del medicamento.

A su vez, diferentes tipos de epilepsia son más propensas a permitir esta interrupción que otras. Diferentes estudios sitúan en $41 \%$ el riesgo de recaída a los 2-5 años, sin embargo, un intento de descontinuación no es seguido de una menor respuesta terapeútica subsecuente, ni se ha logrado objetivizar un mayor riesgo de estatus 
epiléptico, muertes súbitas relacionadas a la epilepsia $u$ otros eventos graves. De nuevo, la decisión de realizar o no un intento de suspensión se debe tomar en conjunto con el paciente de manera individualizada y realizando una ponderación de los riesgos y beneficios para con el mismo ${ }^{27}$.

\section{CONCLUSIONES}

Frente a un paciente con síntomas paroxísticos transitorios, el médico debe considerar una amplia gama de diagnósticos diferenciales, tanto neurológicos como no neurológicos, e inclusive de naturaleza psicogénica. Dentro de estas posibilidades diagnósticas se encuentran los fenómenos convulsivos. La historia clínica, recopilación de datos y examen físico resultan clave en la identificación de dicho fenómeno como una convulsión. Junto a este abordaje, a todo paciente se le debe realizar un electroencefalograma y un estudio de imágenes, preferiblemente resonancia magnética. Existen más estudios a disposición sin embargo ninguno es protocolario y se debe decidir caso a caso su realización.

Una vez identificado el fenómeno como convulsión, esta se debe categorizar como provocada o no provocada, así como sintomática aguda o remota. Además, se debe especificar si la convulsión corresponde a un evento de tipo focal, generalizado o desconocido. Tomando en cuenta la naturaleza de la convulsión y los hallazgos o no que se evidencien en el EEG y resonancia magnética se estima el riesgo de futuras recurrencias, $\mathrm{y}$ en caso de presentar alteraciones en específicas en los estudios mencionados y tratarse de una convulsión no provocada, se procede a catalogar al paciente como epiléptico. Si por otro lado el paciente presenta dichos estudios sin alteraciones relevantes, el riesgo a los 2 años de evidenciar recaídas debe ser comunicado al paciente.

Los tratamientos anticonvulsivantes de índole farmacológica deben ser iniciados en pacientes que cumplan la definición de epilepsia, y abstenerse de brindarlos en convulsiones provocadas y sintomáticas agudas. La decisión de instaurarlos en el caso de una única convulsión no provocada que no cumpla per se con la definición de epilepsia debe ser dialogada con el paciente tomando en cuenta los riesgos y beneficios concretos del mismo, dejando claro que si bien es cierto disminuye la recurrencia en los primeros 2 años, en períodos más extensos este beneficio no se observa y el fármaco no tiene ningún impacto mejorando la calidad de vida.

Por otro lado, estos medicamentos si pueden generar efectos adversos, sin bien es cierto leves y reversible en su mayoría. La suspensión de dicho tratamiento posterior a un período libre de crisis es una posibilidad real la cual por igual debe ser una decisión individualizada y conversada con cada paciente según su perfil de convulsiones, pros y contras.

\section{BIBLIOGRAFÍA}

1. Brumholz K, Wiebe S, Gronseth $\mathrm{G}$ et al. Evidence-Based Guideline: Management of an Unprovoked First seizure in adults. Epilepsy Currents 2015; 15(3): 144-152.

2. Krumholz A, Wiebe S, Gronseth G et al. Practice Parameter: Evaluating an apparent unprovoked first seizure in adults (an evidencebased review). Neurology 2007; 69(21): 19962007.

3. Bergey G. Management of a First 2016;22(1):38-50.

4. Louis E, Cascino G. Diagnosis of Epilepsy and Related Episodic Disorders. Continuum (Minneap Minn) 2016;22(1):15-37.

5. Scheffer I, Berkovic S, Capovilla G. ILAE classification of the epilepsies: Position paper of the ILAE Comission for the Classification and Terminology. Epilepsia 2017; 58(4):512-521.

6. Daroff R, Jankovic J, Mazziotta J et al. Bradley's Neurology in Clinical Practice. Sev- 
enth Edition. Chapter 101: Epilepsies. Elsevier. 2016; 1563-1614.

7. Hauser W, Beghi E. First seizure definitions and worldwide incidence and mortality. Epilepsia 2008; 49(Suppl. 1): 8-12.

8. Hauser L, Stephenson A. Harrison's Neurology in Clinical Medicine. 4th Edition. Chapter 31: Seizures and Epilepsy. McGrawHill Education. 2017;297-322.

9. Fisher RS, Acevedo C, Arzimanoglou A, et al. ILAE official report: a practical clinical definition of epilepsy. Epilepsia 2014;55(4): 475482.

10. Majersik J. Inherited and Uncommon Causes of Stroke. Continuum (Minneap Minn) 2017;23(1):211-237.

11. Manji H, Connolly S, Kitchen N Et al. Manual Oxford de Neurología. Segunda edición. Capítulo 3: Urgencias Neurológicas. Aula Médica. 2016; 78-79.

12. Biller J. Neurología Práctica. Cuarta Edición. Capítulo 6: Enfoque del paciente con convulsiones. Wolters Kluvers. 2011; 51-60.

13. Shibasaki H, Hallett M. The Neurologic Examination Scientific Basis for Clinical Diagnosis. Chapter 24: Paroxysmal and Functional Disorders. Oxford University Press. 2016; 237.

14. ILAE. Epilepsy Imitators. 2017. Obtenido de:

https://www.epilepsydiagnosis.org/epilepsy-

imitators.html

15. Chen D, LaFrance C. Diagnosis and Treatment of Nonepileptic Seizures. Continuum (Minneap Minn) 2016; 22(1):116-131.

16. Kuehn B. New Adult First Seizure Guideline Emphasizes an Individualized Approach. JAMA 2015; 314(2): 111-113.

17. Samuels M, Rooper A. Samuels's Manual of Neurologic Therapeutics. Ninth Edition. Chapter 2: Epilepsy. Wolters Kluwer, 2017; 3776.
18. Maganti RK and Rutecki P. EEG and epilepsy monitoring. Continuum (Minneap Minn). 2013; 19:598-622.

19. Smith SJ. EEG in the diagnosis, classification, and management of patients with epilepsy. Journal of neurology, neurosurgery, and psychiatry. 2005; 76(2):2-7.

20. Siegler J. First Seizure of Life. 2017. Obtenido de: http://brainwaves.me/first-seizureof-life/.

21. Sofat P, Teter B, Kavak K et al. Time interval providing highest yield for initial EEG in patients with new onset seizures. Epilepsy Research. 2016; 229-232

22. Hauser WA, Rich SS, Lee JR et al. Risk of recurrent seizures after two unprovoked seizures. N Engl J Med 1998;338(7): 429-434.

23. Bora I, Seckin B, Zarifoglu $\mathrm{M}$ et al. Risk of recurrence after first unprovoked tonicclonic seizure in adults. J Neurol 1995;242: 157163.

24. Kho K, Lawn D, Dunne W. First seizure presentation. Do multiple seizures within 24 hours predict recurrence? Neurology 2006; 67: 1047-1049.

25. No authors listed. Randomized clinical trial on the efficacy of antiepileptic drugs in reducing the risk of relapse after a first unprovoked tonic-clonic seizure. First Seizure TrialGroup (FIR.S.T. Group). Neurology 1993; 43(3 pt 1):478-483.

26. Marson A, Jacoby A, Johnson A et al. Immediate versus deferred antiepileptic drug treatment for early epilepsy and single seizures: a randomised controlled trial. Lancet 2005; 365(9476):2007-2013.

27. Britton J. Antiepileptic drug therapy: when to start, when to stop. Continuum Lifelong Learning Neurol 2010; 16(3):105-120.

CONFLICTO DE INTERÉS Y/O AGRADECIMIENTOS 
Los autores declaran que no existió ningún conflicto de interés en el presente reporte.
Figura 1. Esquema de abordaje y tratamiento de primera convulsión en pacientes (ver texto para detalles).

Fuente: Elaboración Propia.

EEG: electroencefalograma; RMN: resonancia magnética; ECG: electrocardiograma.

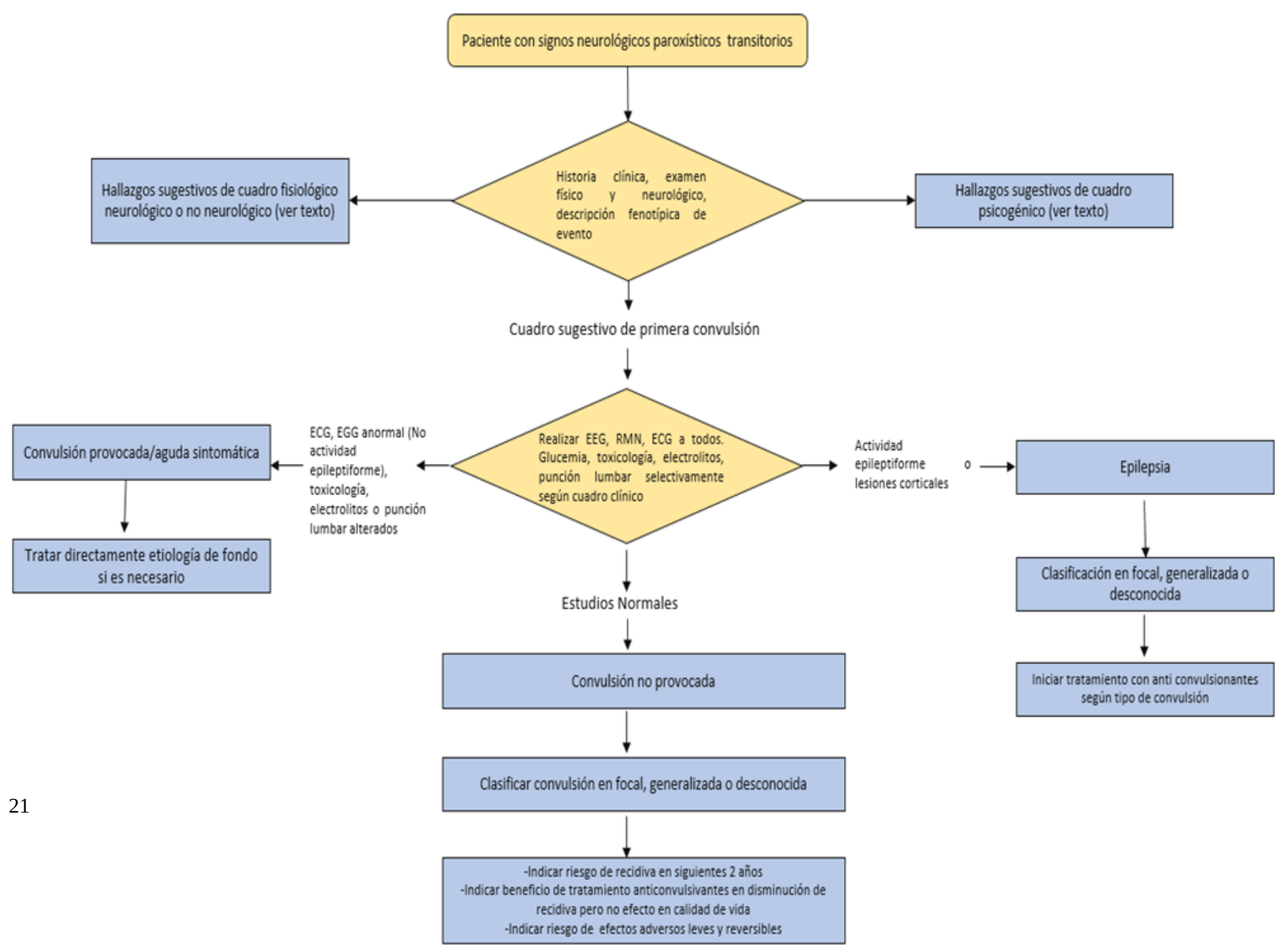

